\title{
Amusias and modularity of musical cognitive processing
}

\author{
Marília Nunes-Silva and Vitor Geraldi Haase \\ Universidade Federal de Minas Gerais, Belo Horizonte, MG, Brazil
}

\begin{abstract}
In the past few years the study of music from a neuroscientific perspective has considerably improved, allowing the evolution of both theoretical knowledge and constructs related to cognitive musical processing. Both neuroimaging studies and studies of individuals who suffer from selective deficits of musical abilities have favored the construction of useful models to understand the mechanisms of musical processing, thus revealing its complexity and eliciting the hypothesis of the modular organization of music in the brain. This article reviews studies of cognitive musical processing with a focus on deficits in musical abilities and the neuropsychological model of cognitive musical processing developed by Isabelle Peretz. This model is an important contribution to neuroscientific studies of music because it furthers the understanding of selective deficits in different components of musical processing that occur in both individuals who incur brain damage and those with congenital amusia. The model also serves as theoretical support for diagnosing different types of amusia. Keywords: amusia, music cognition, music perception, modularity.
\end{abstract}

Received 25 October 2012; received in revised form 07 February 2013; accepted 11 February 2013. Available online 27 June 2013.

\section{Introduction}

Music is not only a social and artistic activity that has been present in all epochs and civilizations but is also a complex cognitive ability. Theoretical, methodological, and empirical contributions from neurology, cognitive psychology, neuropsychology, developmental psychology, and neuroimaging have had a profound impact on music research since the second half of the $20^{\text {th }}$ century, laying the foundation for a neuroanatomical perspective of musical processing. This was made possible because of improved knowledge of the effects of brain damage on musical functioning, the brain mechanisms involved in perception, memory, attention, and musical production, and the areas of the brain that are involved in the processing of different aspects of musical structure (Critchley \& Henson, 1977; Brust, 2001; Peretz \& Zatorre, 2005).

One trend in studies of musical cognitive neuroscience is to explore the neural substrates involved in music perception and performance to understand the biological and functional bases of music (Peretz, 2006). Research shows that musical processing constitutes itself in a specific cognitive domain within specialized neural networks (Zatorre, 2001; Peretz, 2003; Peretz \& Coltheart, 2003).

Marília Nunes-Silva and Vitor Geraldi Haase, Departamento de Psicologia da Universidade Federal de Minas Gerais, Brazil. Correspondence regarding this article should be directed to: Marília Nunes-Silva, Av. Antônio Carlos 6627, FAFICH-UFMG, Sala 2016, Belo Horizonte, MG, CEP 31270-901, Brazil. Phone: +55 (31) 3409-6295, +55 (31) 9209-2826. E-mail: musicainfinita@ig.com.br
In this context, the ability to acquire musical skills with regard to music perception and performance may be seen as an evolutionary adaptation based on natural selection influenced by specific genes. Recent neuropsychological studies directed toward brain specialization in music have investigated congenital disorders in the ability to acquire musical skills (Peretz, 2003). Research on congenital amusia has increased in the last decade and shed new light on the evaluation of musical abilities with regard to education and clinical diagnosis (Peretz, Champod, \& Hyde, 2003).

This article reviews studies of musical ability deficits and both traditional and novel studies of cognitive musical processing that allowed improvements in theoretical constructs and the development of useful models to understand the underlying mechanisms. The cognitive neuropsychological model of musical processing developed by Isabelle Peretz (Peretz et al., 2003) is also presented together with evidence that supports it and reveals its limitations, highlighting the complexity of cognitive musical processing and the modular organization of music in the brain.

\section{Amusias}

Since the last half of the $20^{\text {th }}$ century, neurologists have analyzed disorders in musical function in patients with brain illness in an attempt to associate such lesions with specific brain deficits. Such deficits have been grouped under the term amusia, which was coined by Steinhal in 1871 to generically describe the inability to perceive music (Steinhal, 1871; Wertheim, 1969; Warren, 2004; García-Casares, Torres, Walsh, \& González-Santos, 2011). Amusia was introduced as a 
medical concept that corresponds to musical aphasia by the German physician and anatomist August Knoblauch in 1888 to describe a specific disorder in detecting tones that result from lesions of the motor center (Knoblauch, 1890). Knoblauch (1890) distinguished disorders of music production and comprehension using the terms "amusia" for motor disorders and "tone deafness" and "noteblindness" for sensory disorders. Since then, the concept of amusia was utilized by subsequent authors in a more general sense and has been accepted as indicative of lesions of the right temporal lobe (Henson, 1977). The earliest cognitive model of music with a classification of amusias was also proposed by Knoblauch (1890). Knoblauch proposed a detailed cognitive model of musical processing based on clinical observations of patients and suggested that amusias were a complex and heterogeneous group of disorders of music processing, with possibly nine separate types that reflect impaired music perception and performance (Knoblauch, 1890; Johnson \& Graziano, 2003).

According to Henson (1977), most authors emphasized clinical case studies of amusic individuals, seeking to determine the loci of musical function or studying the relationship between amusia and aphasia rather than classifying amusias. Henson (1977) mentioned important studies that were performed with composers who had brain damage. One of the most famous studies was conducted by Alajouanine (1948) where he evaluated the composer Maurice Ravel, who manifested progressive aphasia associated with a loss of the ability to compose music because of impairment in processing compositional rules. In turn, Luria, Tsvetkova, \& Futer (1965) studied the composer Vissarion Shebalin who had aphasia without amusia caused by stroke.

Benton (1977) established a classification of musical deficits based on clinical observations instead of theoretical principles, combining both local and classificatory perspectives. He recognized the difficulties in understanding the broad spectrum of deficits related to musical processing. Benton (1977) defined amusia as the loss or impairment of musical capacity that results from brain disease. According to the Bentonian proposal, the associated disability could be manifested in several ways: $(i)$ receptive amusia (i.e., the difficulty discriminating melodic patterns, timbres, pitches, and tunes), (ii) musical alexia (i.e., the loss of the ability to read musical notation), (iii) musical amnesia (i.e., failure to recognize tunes that were once familiar to the individual), (iv) rhythm disorders (i.e., difficulty discriminating rhythmic patterns or performing them), (v) vocal or oral-expressive amusia (i.e., loss of the ability to sing, whistle, or hum a tune), (vi) instrumental amnesia or musical apraxia (i.e., loss of the ability to play an instrument without having an associated motor deficit), and (vii) music agraphia (i.e., loss of the ability to copy scores or write down a tune that one has heard).

Most of the classes of amusia proposed by Benton (1977) concerned trained musicians who suffered the loss of ability because of brain damage or disorders.
At the time, studies with listeners without specialized musical formation were very difficult because of the lack of appropriate investigational methodology. Although Benton (1977) identified the heterogeneity of musical disorders and sought to relate them to specific loci in the brain, he did not develop a valid or reliable classification because he did not have at his disposal information processing paradigms or advanced techniques for the study of brain function.

Despite the considerable amount of clinical observations made in the $19^{\text {th }}$ century, systematic and methodologically improved analyses have been used only recently in studies of brain damage that causes specific musical deficits. The subtlest observations of these deficits were only made possible because of considerable improvements in the study of music from a neurocognitive perspective in the last 30 years.

Stewart, von Kriegstein, Warren, \& Griffiths (2006) highlighted the following significant improvements: $(i)$ the evolution of theoretical knowledge and constructs that allowed the development of instruments for the systematic evaluation of music disorders, (ii) greater precision in the examination of brain changes that underlie musical performance disorders, which was made possible by the evolution of neuroimaging techniques that permit the identification of more subtle anatomical changes, and (iii) better knowledge of brain function in typical listeners, mainly from studies that use functional neuroimaging techniques.

Studies of patients with brain damage laid the foundation for a neuroanatomical perspective of musical processing. According to Brust (2001), cases of dissociation in which musical ability is compromised without impairments in language are normally associated with damage in the right hemisphere of the brain. These cases present impaired recognition of sounds as music and a monophonic perception of musical features as meter, tempo, and key, not only in music but also in speech.

Severe deficits in melodic processing with the preservation of verbal ability are usually associated with damage in the auditory area of the temporal lobes, mainly the right superior temporal gyrus (Ayotte, Peretz, Rousseau, Bard, \& Bojanowski, 2000). Lesions of the right frontal lobe do not affect elementary tonal perception but induce similar deficits in pitch discrimination that result from damage to the right superior temporal gyrus. However, difficulties that result from frontal damage are characterized by tonal memory disorder and not perceptual difficulty (Zatorre, 2001). The processing of pitch patterns appears to require an interaction between frontal and temporal areas, especially in the right hemisphere.

Although many studies of these musical deficits have been conducted, the term amusia is still very generic. According to Marin \& Perry (1999), this makes comprehension of the brain mechanisms involved in musical processing a difficult task. Moreover, no consensus has been reached about the classification of the many forms and definitions of this syndrome. Amusia 
has also been referred to as note deafness, tone deafness, tune deafness, and dysmelodia (Peretz, Cummings, \& Dubé, 2007). Marin \& Perry (1999) defined amusia as an acquired clinical disorder in the areas of reading, writing, perception, and musical performance caused by brain damage that is not the result of other more basic sensorial, motor, or cognitive deficits. They also considered the existence of specifically perceptual amusias as those that involve symbolic systems of reading and writing (based on prior knowledge) and others related to vocal performance or motor activities. The traditional neuropsychological classifications (Benton, 1977; Marin \& Perry, 1999) do not have within their definitions congenital or developmental amusia, which shall be discussed later. Amusias may be distinguished as acquired or congenital according to their etiology. Acquired amusias result from disease or brain damage caused by accidents and can impair musical function in many ways, depending on the affected brain area. Congenital amusias, in contrast, are present from birth and may occur as a result of genetic influences (Peretz et al., 2007). Congenital amusias are often associated with impaired tonal processing, and most individuals with congenital amusia are capable of detecting variations in temporal structure (Ayotte, Peretz, \& Hyde, 2002).

In addition to studies of amusic individuals with brain damage that permit the identification of brain areas that are critically involved in the processing of a specific function, structural and functional neuroimaging studies are important for understanding the anatomical and functional correlates of amusias (Tramo, Shah, \& Braida, 2002, Wilson, Pressing, \& Wales, 2002). Neuroimaging studies have revealed which brain areas are potentially recruited for psychological processing in a more general manner and are an important resource for the initial discovery of the relationship between cognitive and neural processes.

Although differences exist between methodological anatomical-clinical correlation paradigms and neuroimaging techniques, results generated by these methods have been consistent and convergent, suggesting the occurrence of selective deficits of components related to musical abilities. Therefore, amusias constitute a complex and heterogeneous group of musical processing disorders, and data simply from anatomical-clinical correlations are insufficient to establish a valid and reliable classification for different types of amusia. In this context, the introduction of the information processing paradigm may complement and structure the knowledge obtained from studies of musical abilities. The model of information processing permits functional interpretations of clinical findings and their neuronal correlates to allow the operationalization of deficits based on neural correlates. The construction of an information processing model for musical stimuli would allow refinement of the classification of different types of amusia and measures of specific components of musical processing.

\section{Cognitive-neuropsychological model of musical perception and memory}

Improvements in the study of music from a cognitive perspective have combined the evolution of theoretical knowledge and constructs related to cognitive musical processing. Studies performed with individuals who present selective deficits in musical abilities caused by brain damage (e.g., Peretz et al., 2003) allowed the construction of useful models to understand amusia and the components involved in musical processing.

According to Peretz et al. (2003), musical memory and perception are basic functions that can be observed and evaluated in all normal listeners, both in musicians and non-musicians. Music perception and musical memory depend on the proper functioning of multiple components and have been investigated in both the cognitive and neuropsychological domains. To evaluate these functions, Peretz et al. (2003) suggested a model that specifies the architecture of the musical system, its components, and its possible interactions or processes. In the cognitiveneuropsychological model of musical processing (Figure 1), damage can impair a processing component (boxes) and interfere with information flux among components (arrows). The model proposes different modules of musical processing, each conceived as an operation of processing particular information that contributes to the entire system (Peretz \& Coltheart, 2003).

In the cognitive-neuropsychological model, the auditory input, which consists of any acoustic stimuli, first passes through an acoustic analysis module in which sound mixtures from different sound sources are segregated. The auditory input relies both on aspects that elicit the language processing action system and aspects that activate the musical processing system, insofar as both systems parallel each other (Peretz et al., 2003). The activation of musical or language processing is determined by aspects of the input to which the module is tuned, although the information proceeds to both domains, but these only respond to information that they were programmed to answer. Song lyrics, therefore, are processed in the language system, represented on the right in the model. The auditory musical input is then analyzed by two parallel and independent systems with specific functions: $(i)$ one system for the melodic dimension (defined by sequential variation in the sound frequency), represented by the contour (i.e., the direction of pitch), scale (i.e., related to tonal functions), and interval (i.e., the size of the frequency interval between tones) and (ii) one system for the temporal dimension (defined by sequential variation in the sound duration), represented by rhythm (i.e., the grouping of events according to temporal proximity) and metric organization (i.e., basic temporal regularity or pulsation). The metric analysis corresponds to spontaneous foot tapping and thus has a direct connection in the model with this output (Peretz \& Coltheart, 2003).

Both pathways and dimensions, melodic and temporal, define the components of musical analysis, sending its respective outputs or a combination of both 


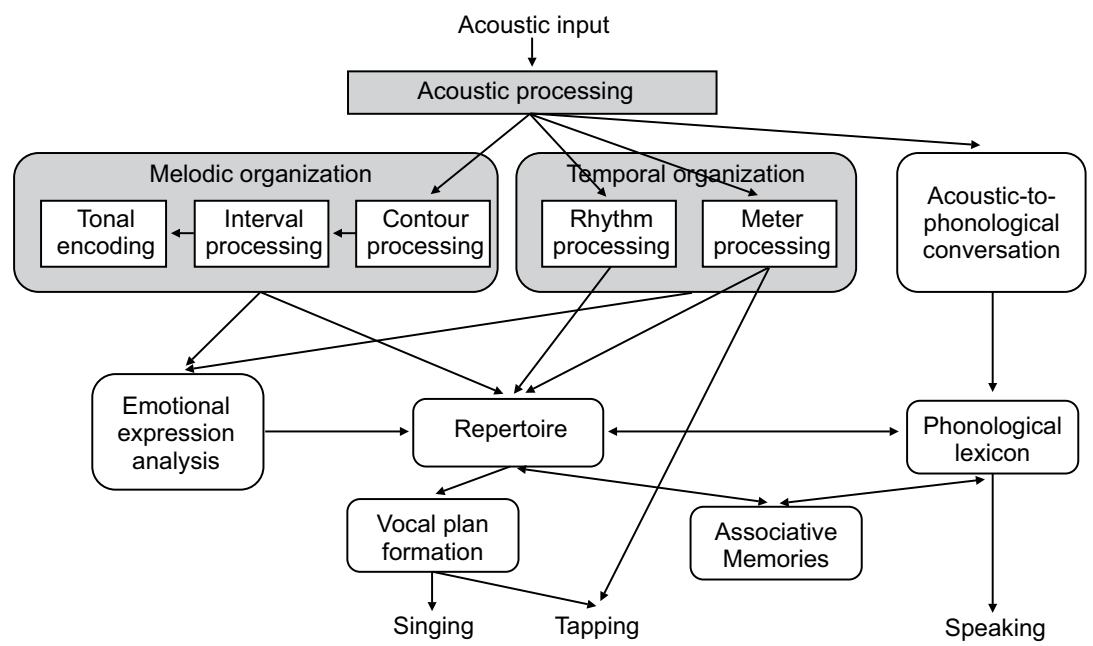

Figure 1. Cognitive-neuropsychological model of music processing, sketching the components and processes involved in music recognition (adapted with permission from Macmillan Publishers Ltd: [Nature Neuroscience] (Peretz, I. \& Coltheart, M. Modularity of music processing. Nature Neuroscience, 6(7), 688-691), copyright (2003).

to a repertoire or component analysis of emotional expression. The repertoire is conceived as a perceptual representation system that contains all representations of musical phrases to which a subject has been exposed during his/her lifetime. The output of the repertoire can activate representations stored in other systems such as lexical representation (in the case of recognizing lyrics) or associative memory (for retrieving and pronouncing the title), leading to music identification. If an individual sings a song, then the corresponding melody represented in the musical lexicon is paired with the associated lyrics that are stored in the phonological lexicon and integrated and planned in such a way that is adequate for vocal performance. It may also activate non-musical information such as an episode related to that song. Successful activation of the repertoire evokes a feeling of familiarity that leads to recognition, even if naming one part of the music is not possible. Therefore, this component appears to be involved in the processing of both familiar music and novel music (Peretz et al., 2003; Peretz \& Coltheart, 2003).

Finally, the emotional component in the model refers to affective information provided by the musical input and depends on two structures: the mode, which is the character of one scale that varies according to the position of tones and semi-tones and its relationship to the tonic, and the tempo, which refers to the music speed (Peretz \& Coltheart, 2003).

The neuropsychological model of cognitive musical processing led to the development of the Montreal Battery of Evaluation of Amusia (MBEA). The MBEA evaluates musical abilities in six components of musical processing: contour, scale, interval, rhythm, meter, and musical memory. It allows the diagnosis of different types of amusia. The MBEA, however, does not evaluate the emotional component present in the model. Therefore, Peretz et al. (2003) suggested the development of tests that evaluate this component so that they may be additionally used in the battery.

\section{Validity of the model}

The components involved in musical processing that are considered in the cognitive-neuropsychological model have been isolated in studies of patients with brain damage who presented deficits in musical abilities. Two parallel and dissociated routes for musical input have been distinguished: temporal and melodic (Peretz, 1990; Peretz \& Kolinsky, 1993; Ayotte et al., 2000; Piccirilli, Sciarma, \& Luzzi, 2000; Vignolo, 2003). When one of these pathways is damaged, the other may be preserved and vice versa, providing evidence of a double dissociation. Peretz $\&$ Zatorre (2005) considered the fact that although the dependence of rhythm and melodic processing is questionable, neuropsychological studies have indicated that melodic and temporal structures are processed independently. Thus, brain damage can produce a selective loss of perception in both the temporal and melodic dimensions.

Other studies highlighted the existence of distinct mechanisms within the melodic dimension: the contour and interval (Peretz, 1990; Ayotte et al., 2000). Damage in the left hemisphere was shown to impair interval processing, without affecting the ability to represent melodies relative to their contour. Damage in the right hemisphere interfered with both processes. Peretz et al. (2003) suggested that damage in the right hemisphere affects the processes necessary for contour representation, depriving intact structures in the left hemisphere of the information required to process interval information.

Peretz \& Zatorre (2005) stated that tonal aspects of melodic processing have been little studied using neuropsychological methods. Nevertheless, evidence suggests that a specialized neural network processes tonal structure (i.e., scales) in melodies. Peretz (1993), for example, analyzed one case of a patient who, after brain damage, acquired a specific deficit in melodic tonal interpretation, although temporal structure and the 
ability to codify music in terms of melodic contour and interval size were preserved.

Dissociations between mechanisms of temporal organization - rhythm and meter - have been investigated in few studies (Peretz, 1990; Liégeois-Chauvel, Peretz, Babaï, Laguitton, \& Chauvel, 1998). These studies showed that meter may be preserved with selective dysfunction of rhythmic structure processing and vice versa. Although more studies are needed, neuropsychological evidence suggests a double dissociation between rhythm and meter. Table 1 presents some studies published from 1990 to 2003 that provided evidence of the modularity of cognitive musical processing.

Considering the emotional component, Peretz \& Gagnon (1999) studied a patient with amusia caused by brain damage who was able to use the mode and tempo of music to make emotional judgments (i.e., determine whether a melody was happy or sad), although the patient could not recognize or process melodic musical information. Peretz \& Gagnon (1999) found that the determinant structure of emotions (e.g., mode and tempo) utilizes pathways that are different from those involved in music recognition.

With regard to music and language, many questions are still unanswered. Based on statements from patients with amusia and without aphasia and vice versa, Peretz et al. (2003) argued for a double-dissociation between musical and language processing. To assess these patients, Peretz et al. (2003) relied on a review developed by Marin \& Perry (1999) in which some cases were grouped. Patel (2003) provided evidence that most of the cases were from the end of the $19^{\text {th }}$ century and beginning of the $20^{\text {th }}$ century and usually involved professional musicians, thus not necessarily providing conclusions that could be extended to the general population. Patel (2003) also stated that case studies of aphasia without amusia, even today, lack systematic tests that evaluate syntactic musical processing with regard to harmonic processing. This author proposed a specific point of contact between syntactic processing in music and language based on neuroimaging data and aspects of cognitive theory.

More recent and systematic studies have been conducted with cases of aphasia without amusia (Warren, Warren, Fox, \& Warrington, 2003) and amusia without impairment of speech and environmental sounds (Peretz et al., 1994; Peretz, Belleville, \& Fontaine, 1997; Piccirilli et al., 2000). Notably, the cases cited by Marin $\&$ Perry (1999) all referred to acquired amusias because the congenital condition has only recently received more attention. Cases of congenital amusia support the differentiation between the cognitive domains of music and language. These individuals present severe musical deficits but maintain their linguistic abilities (Ayotte et al., 2002).

Even if music and language constitute different domains, some similarities exist among some of their perceptual characteristics. In addition to syntactic processing, a likely similarity exists between the processing of melodic contour and speech contour (i.e., prosody). Amusias may be followed by perception deficits with regard to speech intonation, suggesting linkages between rhythmic and melodic patterns in speech and music, and prosody and pitch discrimination may share neural networks (Nicholson, Baum, Kilgour, Koh, Munhall, \& Cuddya, 2003; Patel, Peretz, Tramo, \& Labreque, 1998). Bautista \& Ciampetti (2003) argued that aprosodia, such as amusia, is frequently associated with damage in the right hemisphere. To support this, they presented a case of a woman with expressive amusia and aprosodia who was unable to sing and whose speech sounded monophonic.

Patel, Wong, Foxton, Lochy, \& Peretz (2008) considered amusia to be associated with deficits in prosody. They analyzed two groups in which $\sim 30 \%$ of congenital amusic individuals had difficulties in prosody when discriminating pitch changes that occurred at the end of sentences, implying changes in intention. Such a deficit may be associated with difficulty in discriminating pitch directions with regard to speech, although they found movement among sounds. According to the authors, the fact that perception deficits in pitch direction extensively impact speech perception indicates the need to incorporate prosody perception tests in the diagnosis of amusia to dissociate cases that have purely musical deficits and cases that have both impaired language and musical abilities. They also indicate the need for studies that systematically manipulate stimuli with regard to the distance between sound intervals to compare the discrimination of linguistic pitch direction between amusic individuals and controls.

Peretz (2009) discussed the notion of the modularity of vocal production and provided evidence of modularity in speaking and singing by examining the extent to which vocal production in music and language share processing components. According to Peretz (2009), a double-dissociation exists between singing and speaking. Therefore, domain-specificity must be extended to music and language production tasks, and musical abilities may partially depend on modular processes. Moreover, evidence of pitch-related processes argue against the view that music is anchored in speech module mechanisms.

Considering the above discussion, we argue that musical lyrics are processed in parallel to musical melodies. This hypothesis is supported by Besson, Faïta, Peretz, Bonnel, \& Requin (1998). Amusic individuals can recognize a melody from its lyrics. However, when the lyrics are absent, amusic individuals are unable to identify it. Therefore, in the model proposed by Peretz et al. (2003), lyrics are processed in a parallel manner in a different system (i.e., the language processing system).

Importantly, the model proposed by Peretz et al. (2003) was developed based on patterns of doubledissociations observed in studies of patients with brain damage. The findings that indicate double-dissociations and their implications for the organization of musical function in the brain should be carefully considered. 
Table 1. Case studies of acquired amusias published from 1990 to 2003 showing selective deficits in musical processing

\begin{tabular}{|c|c|c|c|c|c|c|c|c|}
\hline \multirow[b]{2}{*}{ Study } & \multirow[b]{2}{*}{ Cases } & \multirow[b]{2}{*}{ Lesions } & \multicolumn{3}{|c|}{ Melodic organization } & \multicolumn{2}{|c|}{ Temporal organization } & \multirow[b]{2}{*}{ Memory } \\
\hline & & & Scale & Contour & Interval & Rhythm & Meter & \\
\hline \multirow[t]{6}{*}{ Peretz (1990) } & V.G & RH & NA & - & - & + & + & + \\
\hline & V.C. & RH & NA & - & - & + & + & + \\
\hline & L.N. & LH & NA & + & + & - & + & + \\
\hline & C.V. & LH & NA & + & + & - & + & + \\
\hline & 5 cases & RH & NA & - & - & - & + & + \\
\hline & 5 cases & LH & NA & + & - & - & + & + \\
\hline Peretz (1993) & G.L. & Bilateral & - & + & + & + & + & - \\
\hline Peretz \& Kolinsky (1993) & C.N. & Bilateral & - & - & - & + & + & NA \\
\hline \multirow[t]{7}{*}{ Ayotte et al. (2000) } & LBS1 & LH & + & - & + & + & + & - \\
\hline & LBS4 & LH & + & + & + & - & - & - \\
\hline & RBS9 & $\mathrm{RH}$ & - & - & + & + & + & - \\
\hline & $\begin{array}{l}\text { RBS11 } \\
\text { RBS12 }\end{array}$ & RH & + & - & - & + & + & + \\
\hline & BBS20 & Bilateral & - & - & - & + & + & - \\
\hline & N.R. & $\mathrm{RH}$ & - & - & - & - & + & - \\
\hline & R.C. & Bilateral & - & - & - & + & - & - \\
\hline \multirow{8}{*}{$\begin{array}{l}\text { Liégeois-Chauvel et al. } \\
\text { (1998) }\end{array}$} & Case 19 & LH T1p & - & - & - & + & - & + \\
\hline & Case 16 & LH T1p & + & - & - & + & + & - \\
\hline & Case 17 & LH T1p & + & + & - & + & + & - \\
\hline & Case 44 & RH T1p & - & - & - & - & + & - \\
\hline & Case 50 & RH T1p & - & - & - & - & - & - \\
\hline & Case 47 & RH T1p & + & + & + & + & + & + \\
\hline & Case 46 & RH T1p & - & - & - & - & - & + \\
\hline & Case 54 & RH T1p & - & + & - & - & + & - \\
\hline Peretz \& Gagnon (1999) & I.R. & Bilateral & - & - & - & - & + & - \\
\hline Piccirilli et al. (2000) & 1 case & LH (STG) & - & - & - & + & NA & - \\
\hline \multirow[t]{4}{*}{ Vignolo (2003) } & 8 cases & RH & + & + & + & + & + & NA \\
\hline & 2 cases & RH & - & - & - & + & + & NA \\
\hline & 3 cases & LH & + & + & + & - & - & NA \\
\hline & 6 cases & LH & + & + & + & - & - & NA \\
\hline
\end{tabular}

RH, right hemisphere; LH, left hemisphere; T1p, posterior part of superior temporal gyrus; STG, superior temporal gyrus; NA, not assessed.

Van Orden, Pennington, \& Stone (2001) suggested that double-dissociations relate functional behavior to brain lesions and are reference points to identify causal chains in the brain. However, the patterns of dissociation have no meaning if they are outside theoretical guidelines. Modules are a priori assumptions that do not necessarily follow from a double-dissociation. According to Van Orden et al. (2001), the theoretical implications of double-dissociations rely on the validity of modularity. The utility and practicability of assuming that certain modularities are true is questionable. The authors argued that in some cases (e.g., reading modules), modularity fails to converge on a fixed set of exclusionary criteria that define pure case dissociations. Consequently, competing modular theories force continuous pursuits of pure cases, thus increasing the list of exclusionary criteria. This problem partially leads to failures in limiting the potential set of pure case dissociations, which perpetuates further fractionation into more modules. Thus, in addition to questions posed 
by individual differences, reorganization of functions induced by lesions and resource devices must consider the circularity involved in operational definitions of tasks of cognitive components and the subtractive logic implied by pure case dissociations.

Despite this, recent neuroimaging studies have contributed to the validation of double-dissociation findings (Groussard et al., 2010; Salimpoor, Benovoy, Larcher, Dagher, \& Zatorre, 2011). Amusia cases are heterogeneous, affecting either one or more components of cognitive musical processing. Identifying the brain correlates involved in musical processing is difficult. Stewart et al. (2006) reviewed published clinical cases of amusic individuals and functional neuroimaging studies with typical listeners and identified some basic principles that can be used to organize musical processing deficits after brain damage. Despite the fact that the brain damage that caused these deficits was widespread, prevalent damage was found in the right hemisphere. The results suggested some necessary structures for musical processing such as the right superior temporal cortex and other areas such as the insula and frontal lobe. Stewart et al. (2006) considered, however, that the predominance of the right hemisphere in amusias may be attributable to sample bias in which patients with linguistic disorders, usually associated with the left hemisphere, were excluded. Because most cases are the result of stroke, a purely amusia case is rarely found in which musical deficits are followed by other auditory disorders. Nonetheless, Stewart et al. (2006) argued for the possibility of identifying, through dissociations, a few main components of music processing that can be selectively damaged such as pitch, interval, contour, tonal structure, rhythm, meter, timbre, musical memory, and emotion recognition. These components, with the exception of timbre, are included in the model proposed by Peretz et al. (2003). However, the specificity of a brain substrate identified as critical for musical function must still be considered hypothetical. Neuroimaging techniques allow the observation of variations in neural processes that are useful for elucidating neural systems but not yet sufficient to determine the domain of cognitive processing. Using these techniques in an isolated manner is not advisable because they are unable to trace the neural networks that are sufficient to perform a particular cognitive operation. Functional neuroimaging methods and studies of brain-damaged patients may be considered complementary (Price, 2000) and contribute to the identification of brain correlates involved in musical processing.

Importantly, the model proposed by Peretz et al. (2003) refers to the perception of monophonic melodies (i.e., one single voice) and mostly to music perception rather than production. Musical processing has many components and involves the activation of widespread brain areas. Other schemes and models have been proposed based on neuroimaging findings that consider other components such as symbolic system processing, harmony, and timbre (Koelsch \& Siebel,
2005; Warren, 2008; Koelsch, 2011), computational models of music perception and cognition (reviewed by Purwins, Herrera, Grachten, Hazan, Marxer, \& Serra 2008a; Purwins, Herrera, Grachten, Hazan, Marxer, \& Serra, 2008b), and neuroscientific investigations that focus on music production (Bangert \& Altenmüller, 2003; Herrojo-Ruiz, Strübing, Jabusch, \& Altenmüller, 2010; Maidhof, Vavatzanidis, Prinz, Rieger, \& Koelsch, 2010). Nevertheless, the model proposed by Peretz et al. (2003) is a theoretically oriented model with well-established operational definitions that allow the evaluation of musical deficits. Cultural studies that have evaluated amusia using the MBEA indicate that it is a valid and reliable tool in other Western cultures to evaluate musical function (Nunes, Loureiro, Loureiro, \& Haase, 2010; Nunes-Silva \& Haase, 2012). In Eastern cultures that have different rhythm and melody scales, the MBEA stimulus should be adapted according to the specific cultural background for a more sensitive evaluation (Paraskevopoulos, Tsapkini, \& Peretz, 2010). Despite differences in the test stimuli, data obtained by Paraskevopulos et al. (2010) indicated that the cognitive organization of music perception is similar in Greek (i.e., Eastern) and Western cultures. Future studies should identify which cultural aspects are involved in differences in musical perception. In addition to offering support for the assessment of different types of amusia from the MBEA, the model proposed by Peretz et al. (2003), similar to the other proposed models, should advance toward integrating the various aspects of musical production, perception, and cognition into a single framework with well-established neural substrates and considering aspects such as the active nature of perception and developmental and genetic aspects of musical processing.

\section{Congenital Amusia}

According to Hyde \& Peretz (2004), congenital amusia, also known as tone-deafness, is a lifelong inability to process music, despite normal intelligence, memory, and language skills. Individuals with congenital amusia do not develop basic musical abilities, presenting severe deficiencies in tonal processing and difficulties recognizing and distinguishing familiar tunes, distinguishing one tune from another, singing a song, or performing rhythmic patterns. Despite this, these individuals do not present any anomaly or brain damage, and they possess normal levels of education and adequate exposure to musical stimuli throughout life. Baeck (2002) argues that congenital amusic individuals can also recognize prosody, environmental tunes, and human voices, thus characterizing it as a specific musical domain disorder.

The first anecdotal evidence of congenital amusia is attributed to a case described by Charles GrantAllen (1878), which was interestingly followed in the next number of the same journal by a publication of a self-report of the early feminist writer Edith Simcox where she self-declared having the condition previously 
described as well as another similar condition (but not identical) to a case related by Grant-Allen (Simcox \& Grant-Allen, 1878). Despite this, congenital amusia has only recently been systematically evaluated and studied. Ayotte et al. (2002) made one of the first attempts to detail behavioral manifestations in individuals with congenital amusia. They showed that this disorder is part of an entirely new class of learning disabilities that affect musical abilities. Eleven adults participated in the study, which required the self-report of a musical handicap since birth despite much effort to learn music. Self-reports of these individuals were confirmed by a detailed interview and formal testing. These individuals also possessed high levels of education and had no neurological or psychiatric history. The participants were evaluated using a series of three groups of tests: memory recognition tests with musical stimuli, musical pitch perception tests, and musical performance tasks. Most of the tests were originally elaborated for examining the presence of musical deficits in braindamaged patients. Individuals who declared themselves to be amusic were severely impaired in their musical recognition and discrimination abilities compared with controls who were matched with regard to sex, age, level of education, and previous music experience. The amusic participants, for example, could not recognize tunes without help from its lyrics. They were also insensitive to dissonances and could not discriminate differences in pitch. Although these individuals presented such difficulties, most were able to detect variations in temporal structure (Ayotte et al., 2002). Notably, this research focused on the fact that congenital amusia may be related to a deficiency in the processing of variations in pitch frequency. A basic perceptual flaw that compromises pitch frequency in congenital amusia was revealed by Peretz et al. (2002) and confirmed by Hyde \& Peretz (2004). In this research, isotonic and monotonic sequences of five consecutive sounds were presented to amusic individuals and the control group. All amusic individuals had difficulty detecting changes in pitch that were lower than two semi-tones, with normal acuity being approximately a half semi-tone. The affected individuals did not present performance improvement with practice. Conversely, changes in time were detected by the amusic participants the same way as controls, and improved performance was observed with practice.

Many recent studies of congenital amusia have been performed, allowing a better comprehension of these deficits. For example, Hyde, Zatorre, Griffiths, Lerch, \& Peretz (2006) performed a study that compared independent groups (amusic individuals and nonamusic individuals) using magnetic resonance imaging (MRI) data from two different research centers (i.e., Montreal Neurological Institute, McGill University, Montreal, Quebec, Canada and Newcastle University Medical School, Newcastle upon Tyne, UK). They investigated the neural correlates of congenital amusia and hypothesized that there are volumetric differences in both white and gray matter between individuals with and without amusia. The analyses were performed using voxel-based morphometry (VBM), which is a method that allows the investigation of focal differences in brain volume. Hyde et al. (2006) observed a reduction of white matter in the inferior frontal gyrus in amusic individuals compared with non-amusic controls, which was positively correlated with performance on musical tasks based on musical pitch perception but not rhythm perception. The study had one limitation, however, in which VBM only detects macroscopic brain abnormalities, making the detection of subtle brain anomalies impossible. Hyde et al. (2006) expected to find volumetric alterations in the auditory cortex, but such a result was not found. A later MRI study by Hyde, Lerch, Zatorre, Griffiths, Evans, \& Peretz (2007) with the same patients showed that the structural alterations observed with VBM (i.e., a decrease in white matter and increase in gray matter at the level of the inferior right frontal gyrus in amusic individuals) actually reflected an increase in the thickness of the auditory cortex and inferior right frontal gyrus.

Peretz et al. (2007) performed a study of familial aggregation in which they compared families with individuals who suffered from congenital amusia and families with individuals who were all non-amusic. The findings suggested that congenital amusia has a hereditary component that is equivalent to the order of magnitude of heredity found in linguistic impairment. The relative risk for the amusic individuals' siblings and offspring were the following, respectively: $\lambda=$ $10.8,95 \%$ confidence interval, $8-13.5$, and $\lambda=2.3$, $95 \%$ confidence interval, $0-5$. Importantly, Peretz et al. (2007) found differences in the results obtained between the generations (i.e., siblings and offspring). The relative risk for the children of amusic individuals was lower than the relative risk for the siblings. Peretz et al. (2007) suggested that the difference in the relative risk between generations reflected a cohort effect in which congenital amusia could be less penetrating in the children's generation because of a more enriching musical environment. Environmental influences, therefore, contributed to the reduction of the prevalence of congenital amusia in the most recent generation. The authors argued that the importance of environmental factors is paramount and concluded that congenital amusia is likely more influenced by interactions between many genes and the environment, hence favoring the general susceptibility to deficits. Peretz et al. (2007) suggested that congenital amusia is a complex disorder with likely multiple causes that develops epigenetically through interactions between genetic susceptibility factors and variations in individual experiences.

More recent studies of congenital amusia provided evidence of structural and functional alterations in hierarchical processes in the superior temporal area and inferior frontal cortex, with the presence of abnormal connectivity among these areas, indicating that congenital amusia could be a connectivity disorder 
(Hyde et al., 2006; Hyde et al., 2007; Mandell, Schulze, $\&$ Schlaug, 2007). Studies that used event-related potentials supported this hypothesis (Peretz, Brattico, \& Tervaniemi, 2005; Peretz, Brattico, Järvenpää, \& Tervaniemi, 2009). These studies showed that amusic individuals presented an almost normal ability to detect tonal deviances without being conscious of it. Electrical activity in the auditory cortex in amusic individuals was intact, and the electrophysiological alterations that were found were likely located in pathways outside the auditory cortex. The findings suggested that the neural representation of pitch in the brains of amusic individuals is not capable of establishing contact with the knowledge of tonal pitch through the neural frontal auditory pathway.

Other studies that used different evaluation methods reinforce the concept of congenital amusia as a connectivity disorder. Loui \& Schlaug (2009) used diffusion tensor tractography and found white matter abnormalities in the brains of amusic individuals. The amusic individuals presented volume reductions and changes in the structure of the arcuate fasciculus (i.e., the fiber tract that connects the temporal cortex to the inferior frontal cortex), primarily in the right hemisphere. Additionally, Loui, Alsop, \& Schlaug (2009) showed that amusic individuals who presented reductions of arcuate fasciculus volume had impaired performance, with mismatches in music perception and production, assessed by psychophysical tests. Subsequently, Loui, Hohmann, \& Schlaug (2010) used transcranial direct current stimulation and observed a reduction of the accuracy of pitch perception after stimulation of the inferior frontal area and temporal superior area in normal individuals, demonstrating that the intact function and connectivity of the network that connects these two areas are necessary for the efficient neural manipulation of musical stimuli (Loui et al., 2010). Finally, Hyde, Zatorre, \& Peretz (2011) used functional MRI to evaluate participants who listened to melodic sequences of pure tones in which the distance between consecutive tones varied parametrically. They showed that brain activity increased as the distance between the tones increased, even with fine changes, in the right and left auditory cortices of amusic individuals and controls. In contrast, the right inferior frontal gyrus exhibited low activity and evidence of reduced connectivity in the auditory cortex in amusic individuals compared with controls (Hyde et al., 2011). The findings of these studies suggest that preserved bilateral frontotemporal networks are needed for accurate pitch perception and production.

Congenital amusia has generally been associated with pitch deficits. Nonetheless, similar to acquired amusia, congenital amusia can also impair other components of musical processing. Phillips-Silver et al. (2011) recently identified a new case of congenital amusia (Mathieu) related to the ability to perceive the pulsation of music despite normal intelligence and motor and auditory systems. Mathieu was unable to synchronize the movement of his body with music and unable to detect when the movements of a dancer were discordant with the music, although he was able to synchronize with a monometer at a close-to-normal level. Mathieu also presented a score that was under the mean only in the metrics task of the MBEA, indicating selective impairment in this task with preservation of rhythm perception and the perception of pitch frequency patterns. These findings suggested a specific beat deficit in the musical context and that time may have a neurobiological origin distinct from pitch in musical processing.

Studies of congenital amusia have contributed not only to a better understanding of such a disorder but also to a better understanding of musical processing. According to Peretz (2003), studies of congenital amusia may also provide neuropsychological evidence of brain specialization for music, suggesting that the development of neural networks that are dedicated to music occur very early in life and are essential to normal development of musical functions. In congenital amusia, these essential neural elements may be damaged, despite the preservation of other cognitive skills. Further studies are necessary to investigate its etiology and the influence of early musical stimuli on congenital amusia, which may help understand the heritable traits of the disorder and the abilities related to components of cognitive musical processing. The studies of congenital amusia provide evidence that music is a specific domain with specialized neural networks (Peretz, 2006) and suggest the possibility of a very specific type of impairment in the perception of pitch frequency with the preservation of abilities related to temporal structures, consistent with the neuropsychological model of cognitive musical processing.

\section{Conclusion}

Well-documented evidence indicates that musical processing appears to depend on a complex and specific cognitive structure for music according to the hypothesis of the modular organization of music in the brain. According to Fodor (1983), for a system to be considered modular, the following traits must be observed: (i) domain specificity, in which the operations do not cross other domains of content, (ii) innatism (i.e., the extent to which a structure is innate or formed by learning processes, (iii) not assembled (i.e., not formed by collections of more elementary subprocesses, no aggregation), (iv) hardwired (i.e., related to neural specificity, associated with specific, local, and structured neural networks), and (v) autonomous (i.e., not sharing horizontal resources, such as memory and attention, with other cognitive systems). The modular systems must guarantee information speed and automation. According to Fodor, one main trait is informational encapsulation, which indicates that the system does not have complete access to the expectations, beliefs, suppositions, and desires of the individual. Coltheart (1999) suggested that these traits are not absolutely necessary to define the term modular in which one system may be modular but not innate, such as the case 
of the reading system. Coltheart (1999) also indicated that the no-aggregation trait was not well-developed by Fodor and thus inconsistent with his consideration of the presence of top-down processing in the modules. His general idea was that the modules could have sub-levels of representation that communicate among themselves and whose existence, according to Fodor (1983), does not violate the principle of informational encapsulation.

Peretz \& Coltheart (2003) stated that the main trait of a modular system would be the specific domain in which a system only responds to inputs from a particular class. In the case of music, the evidence presented herein indicates that music is constituted in a module of mental information processing whose operations are specific to this type of input, with smaller modules that process domains that are restricted to particular aspects of music. According to Peretz \& Coltheart (2003), musical abilities should not be studied as a general product of cognitive architecture but as a distinct mental module with its own base of knowledge and procedures associated with specific neural substrates. Peretz (2006) stated that studies of the domain specificity of music, innatism, and brain location seek to understand music's functional and biological bases and support the hypothesis of the modular organization of music in the brain. Thus, a neurological anomaly may damage one or more components in processing just as it may interfere with the passage of information among components.

The cognitive-neuropsychological model of musical processing presented by Peretz et al. (2003) may contribute to a better understanding of the selective impairments of different components of musical processing that occur in individuals who suffer brain damage and have congenital amusia. In the clinical classification of amusias presented by Benton (1977), amusias can affect different kinds of musical abilities. The model of musical processing elaborated by Peretz can shed light on the classification proposed by Benton and theoretically support some of these types of amusias including musical amnesia (related to melody recognition), rhythmical disorder (related to the rhythmical component), and receptive amusia (related to the pathway of melodic organization).

Most individuals affected by congenital amusia present deficits in melodic discrimination, despite the preservation of rhythm (Hyde \& Peretz, 2004). The musical processing model addresses differences in the results of melodic structure tasks and temporal structure tasks in congenital amusia and considers the hypothesis of dissociations between the processing of rhythm and melody.

With regard to the diagnoses of different types of amusia, the model offers support for developing evaluation instruments such as the MBEA, which allows a more precise diagnosis of deficits in musical abilities (Peretz et al., 2003). A more complete neuropsychological exam should include evaluations of the components of musical processing considered in the model, not only to identify deficits in musical abilities but also to determine whether interventions based on elements of music can contribute to the treatment of such disorders.

The various manifestations of amusia can be better understood and investigated based on this model. For example, it deals with selective deficits in rhythm, meter, contour, interval, tonal structure, and musical memory and is in accordance with recent neuroscientific knowledge about cognition and music perception. It also provides relevant insights for better understanding of the relationships between music domains and other domains such as emotion and language.

Importantly, according to the model presented by Peretz \& Coltheart (2003), some of its subdomains such as rhythm, meter, tapping, and the emotional component may not be domain-specific. Some questions related to the differentiation of specific components of music and language are still unanswered and require further studies.

Furthermore, the model mostly refers to music perception, the pathways for music production, and the deficits with which they are associated, including vocal production and motor activities, all of which require further research. Because the model focuses on the music perception of normal listeners, without music formation, it fails to consider amusias based on prior knowledge that involves symbolic systems of reading and writing or playing a musical instrument. The issue of the pattern of connectivity between the superior temporal cortex and inferior frontal cortex in the brains of amusic individuals is also not considered.

Studies of music from a neuroscientific perspective are still too recent. Nonetheless, despite its novelty, much progress has been made. The cognitiveneuropsychological model of musical processing has provided several benefits to this field of knowledge, but it can still be enhanced and extended to other types of amusia.

\section{References}

Alajouanine, T. (1948). Aphasia and artistic realization. Brain, 71, 229-241.

Ayotte, J., Peretz, I., \& Hyde, K. (2002). Congenital amusia: A group study of adults afflicted with a music-specific disorder. Brain, 125, 238-251.

Ayotte, J., Peretz, I., Rousseau, I., Bard, C., \& Bojanowski, M. (2000). Patterns of music agnosia associated with middle cerebral artery infarcts. Brain, 123, 1926-1938.

Baeck, E. (2002). The neural networks of music. European Journal of Neurology, 9, 449-456.

Bangert, M., \& Altenmüller, E. O. (2003). Mapping perception to action in piano practice: A longitudinal DC-EEG study. $B M C$ Neuroscience, 4, 26.

Bautista, R. E. D., \& Ciampetti, M. Z. (2003). Expressive aprosody and amusia as a manifestation of right hemisphere seizures. Epilepsia, 44(3), 466-467.

Benton, A. L. (1977). The amusias. In M. Critchley, \& R. A. Henson (Eds.), Music and the brain: Studies in the neurology of music (pp. 378-397). London: Heinemann Medical.

Besson, M., Faïta, F., Peretz, I., Bonnel, A. M., \& Requin, J. (1998). Singing in the brain: Independence of lyrics and tunes. Psychological Science, 9(6), 494-498.

Brust, J. C. M. (2001). Music and neurologist: A historical perspective. Annals of the New York Academy of Sciences, 930, 143-152.

Critchley, M., \& Henson, R. A. (1977). Music and the brain: Studies in the neurology of music. London: Heinemann Medical. 
Coltheart, M. (1999). Modularity and cognition. Trends in Cognitive Sciences, 3(3), 115-120.

Fodor, J. A. (1983). The modularity of mind: An essay on faculty psychology. Cambridge, MA: MIT Press.

García-Casares, N., Torres, M. L. B., Walsh, S. F., \& González-Santos, M. L. (2011). Modelo de cognición musical y amusia. Neurología, $28,179-186$

Grant-Allen, C. (1878). Note-deafness. Mind, 3(10), 157-167.

Groussard, M., Viader, F., Hubert, V., Landeau, B., Abbas, A., Desgranges, B., ... Platel, H. (2010). Musical and verbal semantic memory: Two distinct neural networks? Neuroimage, 49, 27642773.

Henson, R. A. (1977). Neurological aspects of musical experience. In M. Critchley, \& R. A. Henson (Eds.). Music and the brain: Studies in the neurology of music (pp. 3-21). London: Heinemann Medical.

Herrojo-Ruiz, M., Strübing, F., Jabusch, H. C., and Altenmüller, E. (2010). EEG oscillatory patterns are associated with error prediction during music performance and are altered in musician's dystonia. Neuroimage, 55(4), 1791-1803.

Hyde, K., Lerch, J., Zatorre, R., Griffiths, T. D., Evans, A., \& Peretz, I. (2007). Cortical thickness in congenital amusia: When less is better than more. Journal of Neuroscience, 47, 13028-13032.

Hyde, K., \& Peretz, I. (2004). Brains that are out of tune but in time. Psychological Science, 15(5), 356-360.

Hyde, K. L., Zatorre, R. J., Griffiths, T. D., Lerch, J. P., \& Peretz, I. (2006). Morphometry of the amusic brain: A two-site study. Brain, $129,2562-2570$.

Hyde, K., Zatorre, R. J., \& Peretz, I. (2011). Functional MRI evidence of an abnormal neural network for pitch processing in congenital amusia. Cerebral Cortex, 21(2), 292-299.

Johnson, J. K., \& Graziano, A. B. (2003). August Knoblauch and amusia: A nineteenth-century cognitive model of music. Brain and Cognition, 51, 102-114.

Knoblauch, A. (1890). On disorders of the musical capacity from cerebral disease. Brain, 13, 317-340.

Koelsch, S. (2011). Toward a neural basis of music perception: A review and updated model. Frontiers in Auditory Cognitive Neuroscience, 2, 110.

Koelsch, S., \& Siebel, W. A. (2005). Towards a neural basis of music perception. Trends in Cognitive Sciences, 9, 578-584.

Liégeois-Chauvel, C., Peretz, I., Babaï, M., Laguitton, V., \& Chauvel, P. (1998). Contribution of different cortical areas in the temporal lobes to music processing. Brain, 121, 1853-1867.

Loui, P., Alsop, D., \& Schlaug, G. (2009). Tone deafness: A new disconnection syndrome? Journal of Neuroscience, 29(33), 1021510220.

Loui, P., \& Schlaug, G. (2009). Investigating musical disorders with diffusion tensor imaging: A comparison of imaging parameters. Annals of the New York Academy of Sciences, 1169, 121-125.

Loui, P., Hohmann, A., \& Schlaug, G. (2010). Inducing disorders in pitch perception and production: A reverse-engineering approach. Proceedings of Meetings in Acoustics, 9, 050002.

Luria, A. R., Tsvetkova, L. S., \& Futer, D. S. (1965). Aphasia in a composer (V. G. Shebalin). Journal of Neurological Science, 2, 288-292.

Maidhof, C., Vavatzanidis, N., Prinz, W., Rieger, M., \& Koelsch, S. (2010). Processing expectancy violations during music performance and perception: An ERP study. Journal of Cognitive Neuroscience, 22(10), 2401-2413.

Mandell, J., Schulze, K., \& Schlaug, G. (2007). Congenital amusia: An auditory-motor feedback disorder? Restorative Neurology and Neuroscience, 25, 323-334.

Marin, O., \& Perry, D. (1999). Neurological aspects of music perception and performance. In D. Deutsch (Ed.), The psychology of music (pp. 653-712). San Diego: Academic Press.

Nicholson, K. G., Baum, S., Kilgour, A., Koh, C. K., Munhall, K. G., \& Cuddya, L. L. (2003). Impaired processing of prosodic and musical patterns after right hemisphere damage. Brain and Cognition, 52, 382-389.

Nunes-Silva, M., \& Haase, V. G. (2012). Montreal Battery of Evaluation of Amusia: Validity evidence and norms for adolescents in Belo Horizonte, Minas Gerais, Brazil. Dementia \& Neuropsychology, 6(4), 244-252.

Nunes, M., Loureiro, C. M. V., Loureiro, M. A., \& Haase, V. G. (2009). Tradução e validação de conteúdo de uma bateria de testes para avaliação de Amusia. Avaliação Psicológica, 9(2), 211-232.

Paraskevopoulos, E., Tsapkini, K., \& Peretz, I. (2010). Cultural aspects of music perception: Validation of a Greek version of the Montreal
Battery of Evaluation of Amusias. Journal of the International Neuropsychological Society, 16, 695-704.

Patel, A. D. (2003). Language, music, syntax and the brain. Nature Neuroscience, 6(7), 674-681.

Patel, A. D., Peretz, I., Tramo, M., \& Labreque, M. (1998). Processing prosodic and musical patterns: A neuropsychological investigation. Brain and Language, 61, 123-144.

Patel, A., Wong, M., Foxton, J., Lochy, A., \& Peretz, I. (2008). Speech intonation perception deficits in musical tone deafness (congenital amusia). Music Perception, 25, 357-368.

Peretz, I. (1990). Processing of local and global musical information in unilateral brain-damaged patients. Brain, 113, 1185-1205.

Peretz, I. (1993). Auditory atonalia for melodies. Cognitive Neuropsychology, 10(1), 21-56.

Peretz, I. (2003). Brain specialization for music: New evidence from congenital amusia. In I. Peretz, \& R. J. Zatorre (Eds.), The cognitive neuroscience of music (pp. 192-203). Oxford: Oxford University Press.

Peretz, I. (2006). The nature of music from a biological perspective. Cognition, 100, 1-32.

Peretz, I. (2009). Music, language and modularity framed in action. Psychologica Belgica, 49(2-3), 157-175.

Peretz, I., Ayotte, J., Zatorre, R. J., Mehler, J., Ahad, P., Penhune, V. B., \& Jutras, B. (2002). Congenital amusia: A disorder of finegrained pitch discrimination. Neuron, 33, 185-191.

Peretz, I., Belleville, S., \& Fontaine, F. S. (1997). Dissociations entre musique et langage après atteinte cérébrale: Un nouveau cas d'amusie sans aphasie. Revue Canadienne de Psychologie Expérimentale, 51(4), 354-367.

Peretz, I., Brattico, E., Järvenpää, M., \& Tervaniemi, M. (2009). The amusic brain: In tune, out of key, and unaware. Brain, 132, 1277-1286.

Peretz, I., Brattico, E., \& Tervaniemi, M. (2005) Abnormal electrical brain responses to pitch in congenital amusia. Annals of Neurology, 58, 478-482.

Peretz, I., Champod, A. S., \& Hyde, K. (2003). Varieties of musical disorders: The Montreal Battery of Evaluation of Amusia. Annals of the New York Academy of Sciences, 999, 58-75.

Peretz, I., \& Coltheart, M. (2003). Modularity of music processing. Nature Neuroscience, 6(7), 688-690.

Peretz, I., Cummings, S., \& Dubé, M. P. (2007). The genetics of congenital amusia (tone deafness): A family-aggregation study. American Journal of Human Genetics, 81, 582-588.

Peretz, I., \& Gagnon, L. (1999). Dissociation between recognition and emotional judgment for melodies. Neurocase, 5, 21-30.

Peretz, I., \& Kolinsky, R. (1993). Boundaries of separability between melody and rhythm in music discrimination: A neuropsychological perspective. Quarterly Journal of Experimental Psychology, 46, 301-325.

Peretz, I., Kolinsky, R., Tramo, M., Labrecque, R., Hublet, C., Demeurisse, G., \& Belleville, S. (1994). Functional dissociations following bilateral lesions of auditory cortex. Brain, 117, 1283-1302.

Peretz, I., \& Zatorre, R. J. (2005). Brain organization for music processing. Annual Review of Psychology, 56, 89-114.

Phillips-Silver, J., Toiviainenc, P., Gosselina, N., Pichéa, O., Nozaradana, S., Palmera, C., \& Peretz, I. (2011). Born to dance but beat deaf: A new form of congenital amusia. Neuropsychologia, 49, 961-969.

Piccirilli, M., Sciarma, T., \& Luzzi, S. (2000). Modularity of music: Evidence from a case of pure amusia. Neurology, Neurosurgery, and Psychiatry, 69, 541-545.

Price, C. J. (2000). The anatomy of language: Contributions from functional neuroimaging. Journal of Anatomy, 197, 335-359.

Purwins, H., Herrera, P., Grachten, M., Hazan, A., Marxer, R., \& Serra, X. (2008a). Computational models of music perception and cognition: I. The perceptual and cognitive processing chain. Physics of Life Reviews, 5, 151-168.

Purwins, H., Herrera, P., Grachten, M., Hazan, A., Marxer, R., \& Serra, X. (2008b). Computational models of music perception and cognition. II. Domain-specific music processing. Physics of Life Reviews, 5, 169-182.

Salimpoor, V. N., Benovoy, M., Larcher, K., Dagher, A., \& Zatorre, R. J. (2011). Anatomically distinct dopamine release during anticipation and experience of peak emotion to music. Nature Neuroscience, 14(2), 257-262.

Simcox, E. \& Grant-Allen, C. (1878). Note-Deafness. Mind, 3 (11), 401-404.

Steinthal,H.(1871). Einleitung in diePsychologieund Sprachwissenschaft. Abriss der sprachwissenschaft. Berlin: Dümmlers. 
Stewart, L., von Kriegstein, K., Warren, J. D., \& Griffiths, T. D. (2006). Music and the brain: Disorders of musical listening. Brain, $129,2533-2553$.

Tramo, M. J., Shah, G. D., \& Braida, L. D. (2002). Functional role of auditory cortex in frequency processing and pitch perception. Journal of Neurophysiology, 87, 122-139.

Van Orden, G. C., Pennington, B. F., \& Stone, G. O. (2001). What do double dissociations prove? Cognitive Science, 25, 111-172.

Vignolo, L. A. (2003). Music agnosia and auditory agnosia: Dissociations in stroke patients. Annals of the New York Academy of Sciences, 999, 50-57.

Warren, J. (2004). The amusias. In: F. C. Rose (Ed.), Neurology of the arts: Painting, music, and literature (pp. 275-305). London: Imperial College Press.
Warren, J. (2008). How does the brain process music? Clinical Medicine, 8(1), 32-36.

Warren, J. D., Warren, J. E., Fox, N. C., \& Warrington, E. K. (2003). Nothing to say, something to sing: Primary progressive dynamic aphasia. Neurocase, 9(2), 140-155.

Wertheim, N. (1969). The amusias. In P. J. Vinken, \& G. W. Bruyn (Eds.), Disorders of speech, perception and symbolic behaviour (series title: Handbook of clinical neurology, vol. 4) (pp. 195-206). Amsterdam: New Holland.

Wilson, S. J., Pressing, J. L., \& Wales, R. J. (2002). Modelling rhythmic function in a musician post-stroke. Neuropsychologia, 40, 494-505.

Zatorre, R. J. (2001). Neural specializations for tonal processing. Annals of the New York Academy of Sciences, 930, 193-210. 